\title{
ТЕСТИРОВАНИЕ ПРОДУКТОВ
}

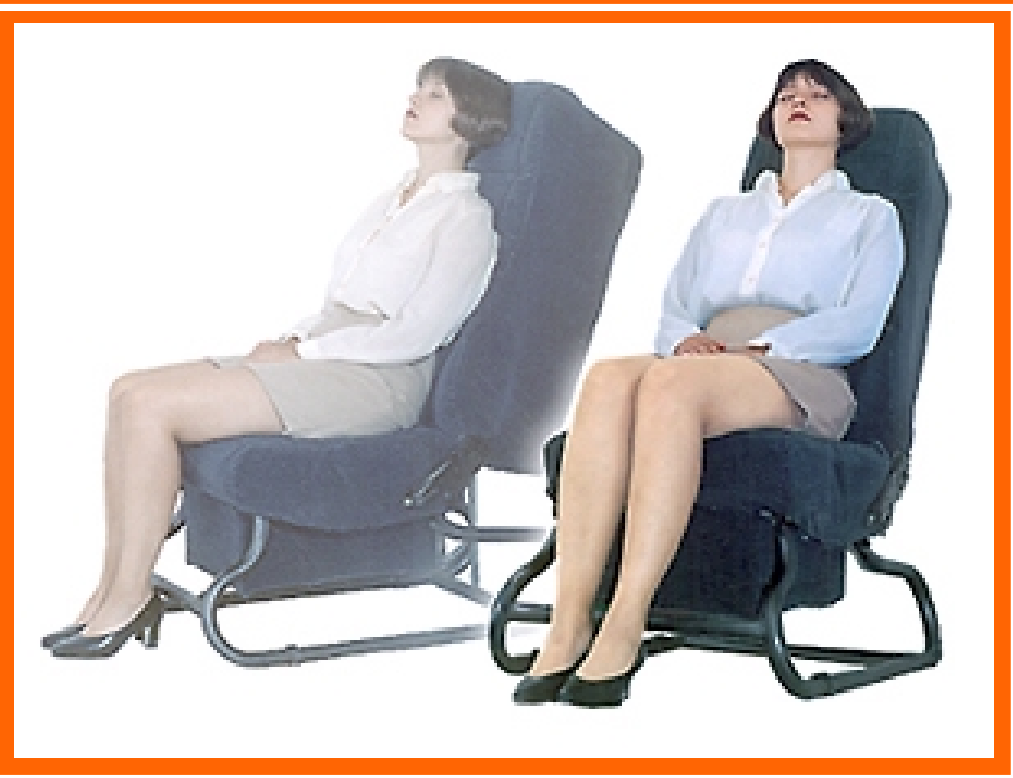


Вместо того, чтобы делать то, что вы делали всегда и потом пытаться это продать, узнайте, что будет продаваться и сделайте это. 
ЧТО ТАКОЕ ТЕСТИРОВАНИЕ ПРОДУКТОВ? PRODUCT TESTING

ТЕСТИРОВАНИЕ ПРОДУКТОВ - ГРУППА МЕТОДОВ ОЦЕНКИ И ДИАГНОСТИКИ ХАРАКТЕРИСТИК ПРОДУКТОВ С ПОМОЩЬЮ ПОТРЕБИТЕЛЕЙ.

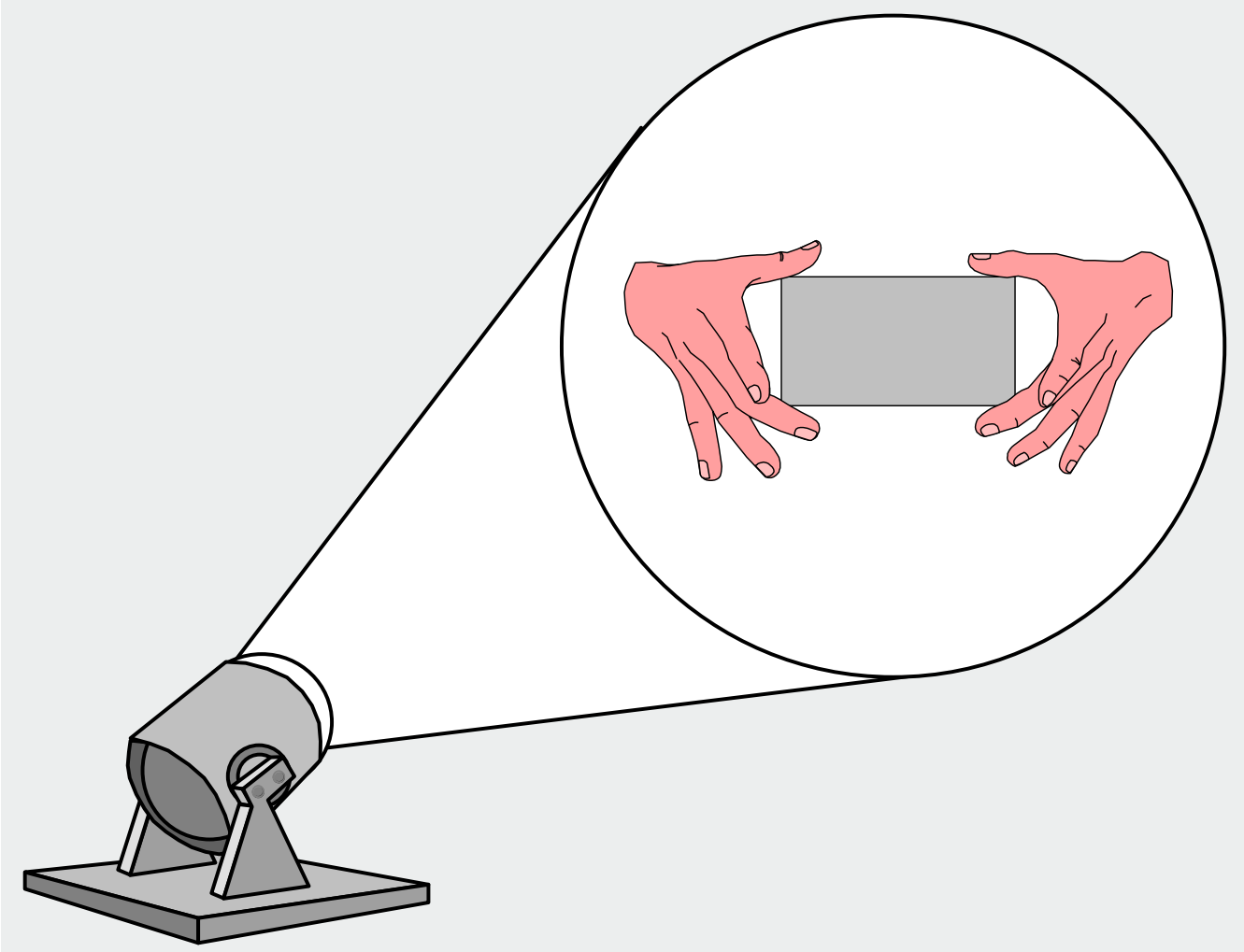
ПРЕДТЕЧАМИ МЕТОДОВ ТЕСТИРОВАНИЯ ПРОДУКТОВ БЫЛИ ТЕСТЫ ЧУВСТВЕННЫХ ВОСПРИЯТИЙ ИЛИ СЕНСОРНЫЕ TECTЫ (SENSORY TESTS) И ПАНЕЛИ ТЕСТЕРОВ (EMPLOYEE PANELS). 
КОГДА ИСПОЛЬЗУЕТСЯ?

- МЕТОДЫ ТЕСТИРОВАНИЯ ПРОДУКТОВ ПРИМЕНЯЮТСЯ ЛИБО:

> ПОСЛЕ ТОГО, КАК СКРИНИНГ/ТЕСТИРОВАНИЕ КОНЦЕПЦИЙ (CONCEPT SCREENING) ПОЗВОЛИЛ ВЫЯВИТЬ ВЫИГРЫШНУЮ ИДЕЮ ПРОДУКТА,

> НА СТАДИИ РАЗРАБОТКИ ПРОДУКТА, КОГДА В РЕЗУЛЬТАТЕ НИОКР, СЕНСОРНЫХ ТЕСТОВ/ ПАНЕЛИ ТЕСТЕРОВ ВЫЯВЛЯЕТСЯ НОВЫЙ ПРОДУКТ/ВАРИАНТ ПРОДУКТА,

> В ЛЮБОЙ МОМЕНТ ЖИЗНЕННОГО ЦИКЛА ПРОДУКТА, КОГДА НЕОБХОДИМО ОЦЕНИТЬ РЕАКЦИЮ ПОТРЕБИТЕЛЕЙ НА МОДИФИКАЦИЮ ПРОДУКТА (ИЗМЕНЕНИЕ ДИЗАЙНА, СМЕНУ КОМПОНЕНТОВ, СНИЖЕНИЕ ЦЕНЫ И Т.Д.)

> В ЦЕЛЯХ ПРЕДЪЯВЛЕНИЯ ИСКОВ КОНКУРЕНТАМ. 
ЧТО ТАКОЕ НОВЫЙ ПРОДУКТ?

- НОВЫМ ПРОДУКТОМ МОЖЕТ СЧИТАТЬСЯ:

$>$ ИННОВАЦИОННЫЙ ПРОДУКТ (SONY WALKMAN)

> РАСШИРЕНИЕ ЛИНЕЙКИ («БАЛТИКА 0», «БАЛТИКА 8»)

$>$ КЛОН ИЛИ «ФЛАНГОВЫЙ» ПРОДУКТ (АСПИРИН UPSА/УПСАРИН, КОПИРОВАЛЬНЫЙ АППАРАТ RICOH versus XEROX)

$>$ ПЕРЕПОЗИЦИОНИРОВАНИЕ СУЩЕСТВУЮЩИХ ТОВАРОВ (СЕТЬ

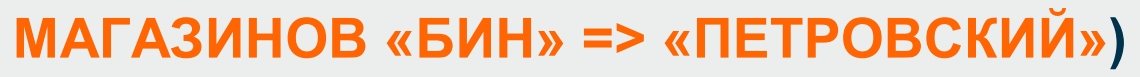

> НОВАЯ ФОРМЫ СУЩЕСТВУЮЩИХ ТОВАРОВ (ЖИДКОЕ МЫЛО)

> НОВАЯ ФОРМУЛА СУЩЕСТВУЮЩИХ ТОВАРОВ (КОКА КОЛА)

> НОВАЯ УПАКОВКА СУЩЕСТВУЮЩИХ ТОВАРОВ (ПЛАСТИКОВАЯ БУТЫЛКА ПЕПСИ 0.6Л ВМЕСТО 0.5Л)

> ПРОДУКТЫ ДОБАВЛЯЮЩИЕ ЦЕННОСТЬ (ЛОСЬОН «ЖИЛЕТТ»)

> ВРЕМЕННЫЕ/ПРОМЕЖУТОЧНЫЕ ВАРИАНТЫ ПРОДУКТОВ (WINDOWS MЕ) 


\section{ПРОЦЕДУРА ТЕСТИРОВАНИЯ ПРОДУКТА (ВАРИАНТ)}

ПРЕЗЕНТАЦИЯ ПРОДУКТА/ЦЕНЫ

\

ПРОБА НАМЕРЕНИЕ КУПИТЬ И

ОБЩАЯ ОЦЕНКА ПРОДУКТА

凡

(ПРЕДПОЛАГАЕМАЯ/ОБЫЧНАЯ) ЧАСТОТА ПОКУПКИ, ПОКУПКА ВМЕСТО ... (ИЛИ "КАННИБАЛИЗАЦИЯ)

$\checkmark$

ИЗУЧЕНИЕ ЦЕНОВОЙ ЧУВСТВИТЕЛЬНОСТИ

凤

СПЕЦИФИЧЕСКИЕ ТРЕБОВАНИЯ К ПРОДУКТУ 


\section{ПРОЦЕДУРА ТЕСТИРОВАНИЯ ПРОДУКТА (ВАРИАНТ)}

ИСПОЛЬЗОВАНИЕ ПРОДУКТА?

?

ОБЩАЯ ОЦЕНКА ПРОДУКТА

?

НАМЕРЕНИЕ ПОВТОРНОЙПОКУПКИ

$\sqrt{2}$

ПОТРЕБИТЕЛЬСКИЕ ПРЕДПОЧТЕНИЯ, ПОЗИЦИЯ ПРОДУКТА СРЕДИ ДРУГИХ

?

ОЦЕНКА ОТДЕЛЬНЫХ ХАРАКТЕРИСТИК ТОВАРА

СПЕЦИАЛЬНЫЕ ВОПРОСЫ 
ОБРАЗЦЫ

- ИСПОЛЬЗУЕМЫЕ В МЕТОДАХ ТЕСТИРОВАНИЯ ПРОДУКТОВ ОБРАЗЦЫ МОГУТ САМЫМИ РАЗНООБРАЗНЫМИ В ЗАВИСИМОСТИ ОТ ЦЕЛЕЙ ТЕСТИРОВАНИЯ И СТАДИИ РАЗРАБОТКИ ПРОДУКТА. ДИАПАЗОН ПРОСТИРАЕТСЯ ОТ КОНЦЕПТУАЛЬНЫХ НЕФУНКЦИОНАЛЬНЫХ МАКЕТОВ ДО ПОЛНОФУНЦИОНАЛЬНЫХ БРЭНДИРОВАННЫХ ПРОДУКТОВ, ТЕСТИРУЕМЫХ В РЕАЛЬНЫХ УСЛОВИЯХ ЭКСПЛУАТАЦИИ.

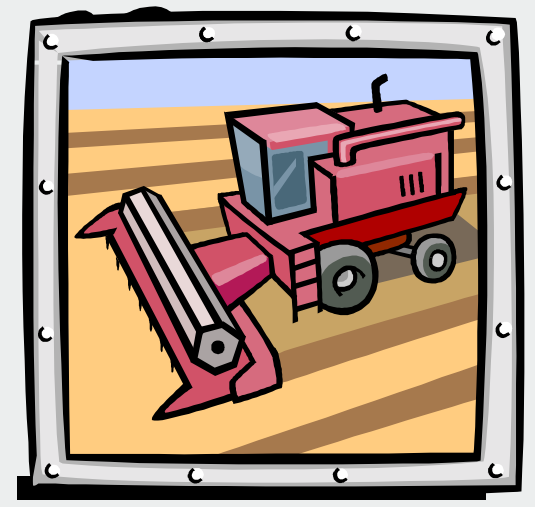


ТИПЫ ТЕСТОВ

- СУЩЕСТВУЕТ ДВА БАЗОВЫХ ПРИНЦИПА ТЕСТИРОВАНИЯ ПРОДУКТОВ:

ПОКАЗ ОДНОГО ПРОДУКТА

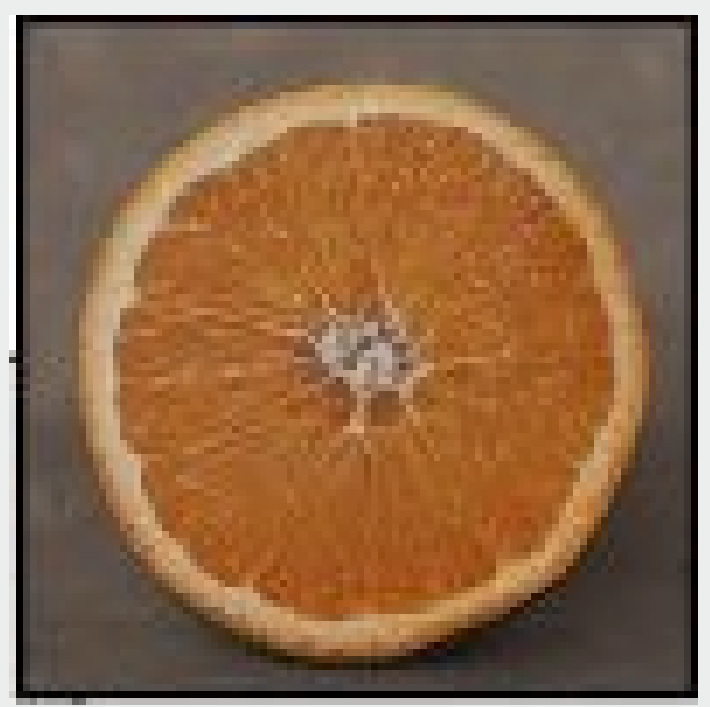

ПРИНЦИП СРАВНЕНИЯ 2-Х И БОЛЕЕ ПРОДУКТОВ
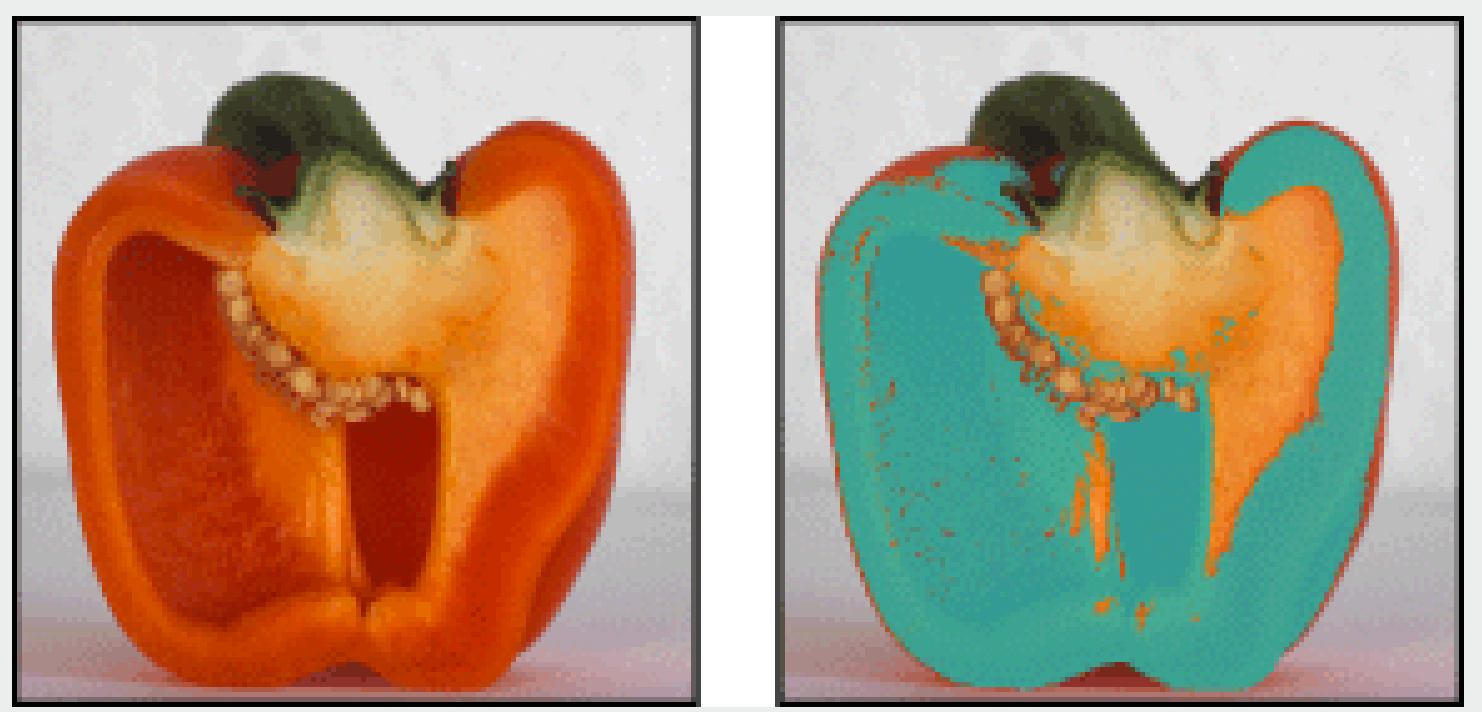
ЕЩЕ ОДНА КЛАССИФИКАЦИЯ ТЕСТОВ

- ЧТО ИЗУЧАЕМ?

1. РАЗНИЦУ (TRIANGLE, DUO-TRIO)

2. ПРЕДПОЧТЕНИЕ (PAIRED COMPARISON, RANKING)

3. ПРИЗНАНИЕ/ПРИНЯТИЕ (HEDONIC, АТTRIBUTE RATONG/SCALING)

4. ДЕСКРИПТИВНЫЙ TЕСТ (QUANTITATIVE DESCRIPTIVE ANALYSIS, PROFILING) 
7 ШАГОВ ПРИ ТЕСТИРОВАНИИ ГИПОТЕЗ

1. СФОРМУЛИРУЙТЕ НУЛЕВУЮ ГИПОТЕЗУ

2. СФОРМУЛИРУЙТЕ АЛЬТЕРНАТИВНУЮ ГИПОТЕЗУ

3. УСТАНОВИТЕ УРОВЕНЬ ЗНАЧИМОСТИ $\alpha$

4. УСТАНОВИТЕ ОБЛАСТЬ ПРИНЯТИЯ/ОТБРАСЫВАНИЯ ГИПОТЕЗЫ

5. ПО ПОЛУЧЕННЫМ В ТЕСТЕ ДАННЫМ ВЫЧИСЛИТЕ НАБЛЮДАЕМЫЕ СТАТИСТИКИ

6. ИНТЕРПРЕТИРУЙТЕ ВЫЧИСЛЕНИЯ

7. СДЕЛАЙТЕ ЗАКЛЮЧЕНИЕ 
ПРЕДУПРЕЖДЕНИЕ

- УРОВЕНЬ ЗНАЧИМОСТИ НЕ ПОКАЗЫВЕТ ВЕЛИЧИНУ РАЗНИЦЫ МЕЖДУ ДОЛЯМИ, СРЕДНИМИ И Т.П.

\section{КОНТРОЛЬ vs А $5 \%$ КОНТРОЛЬ vs В $1 \%$}

- НЕКОТОРЫЕ ПЫТАЮТСЯ СКАЗАТЬ ЧТО В БОЛЕЕ ЗНАЧИМО, ЧЕМ А HO

- 1\% ОЗНАЧАЕТ ТОЛЬКО ТО, ЧТО ВЕРОЯТНОСТЬ НЕПРАВИЛЬНОГО РЕШЕНИЯ МЕНЬШЕ (НАПРИМЕР, ОТВЕРГНУТЬ НУЛЕВУЮ ГИПОТЕЗУ, ЕСЛИ ОНА ВЕРНА) 
ПРИНЯТИЕ ГИПОТЕЗЫ

\section{ДВА ТИПА ОШИБОК}

ОШИБКА ПЕРВОГО РОДА - ТУре I

ОШИБКА ВТОРОГО РОДА - Туре II

МОЩНОСТЬ КРИТЕРИЯ - Power 
ОШИБКА ПЕРВОГО РОДА - Туре I error

\section{в том случае, когда мы ОТВЕРГАЕМ НУЛЕВУЮ ГИПотезУ и оНа явЛяется ВЕРНОЙ}


ОШИБКА ВТОРОГО РОДА - Туре I I error

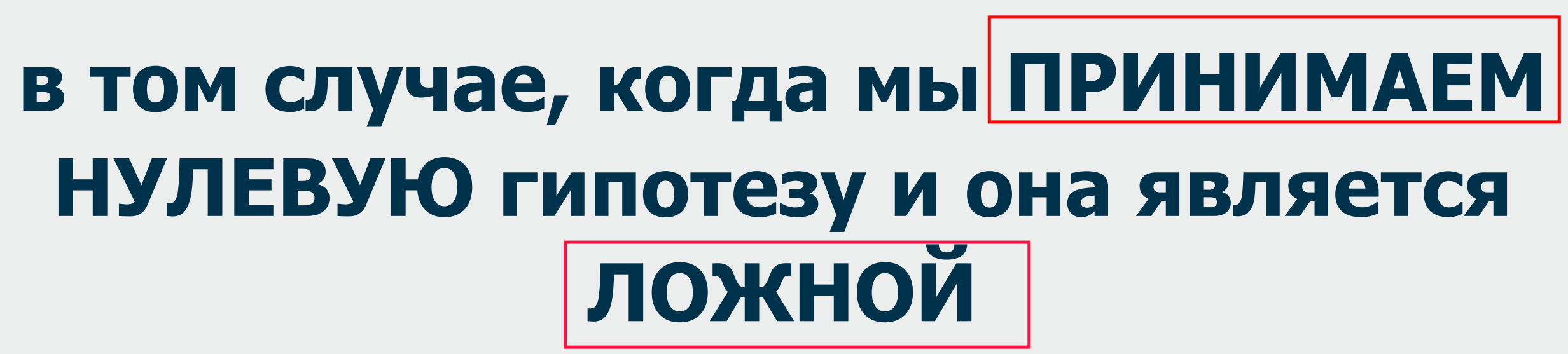


МОЩНОСТЬ КРИТЕРИЯ - POWER

\section{в том случае, когда мы ОТВЕРГАЕМ НУЛЕВУЮ ГиПотезУ и она является ложной}


ТАБЛИЦА ИСТИННОСТИ

\section{КАК В ВЫБОРКЕ?}

\section{KAK B ГC?}

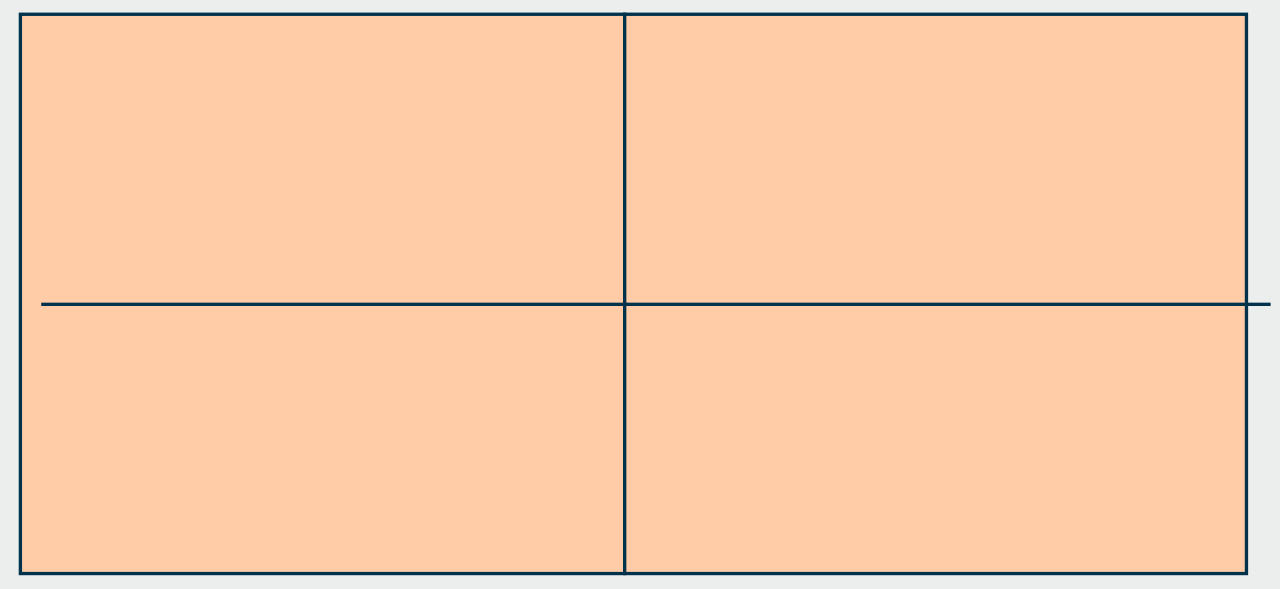


ТАБЛИЦА ИСТИННОСТИ - TRUTH TABLE

\section{В ГЕНЕРАЛЬНОЙ СОВОКУПНОСТИ:}

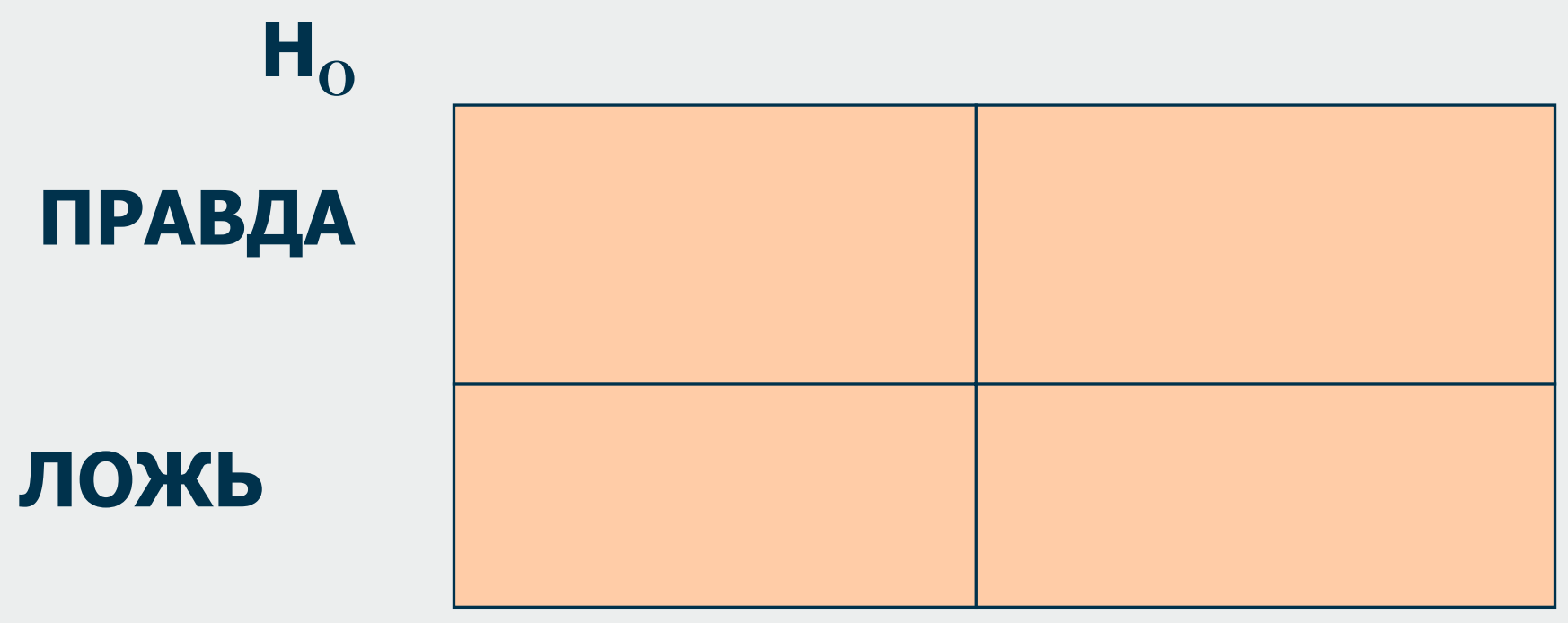


ТАБЛИЦА ИСТИННОСТИ - TRUTH TABLE

\section{ВЫБОРКА}

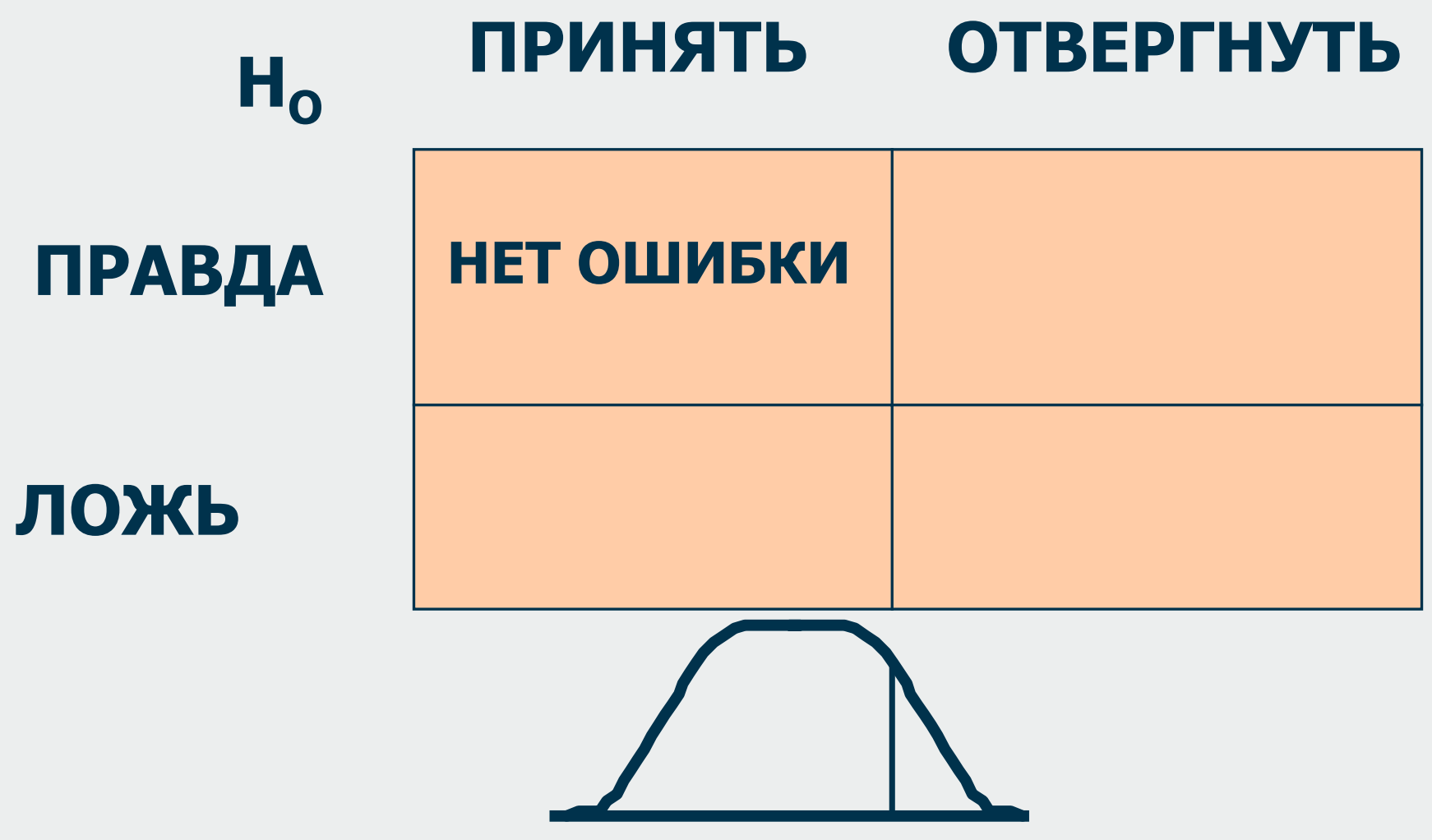




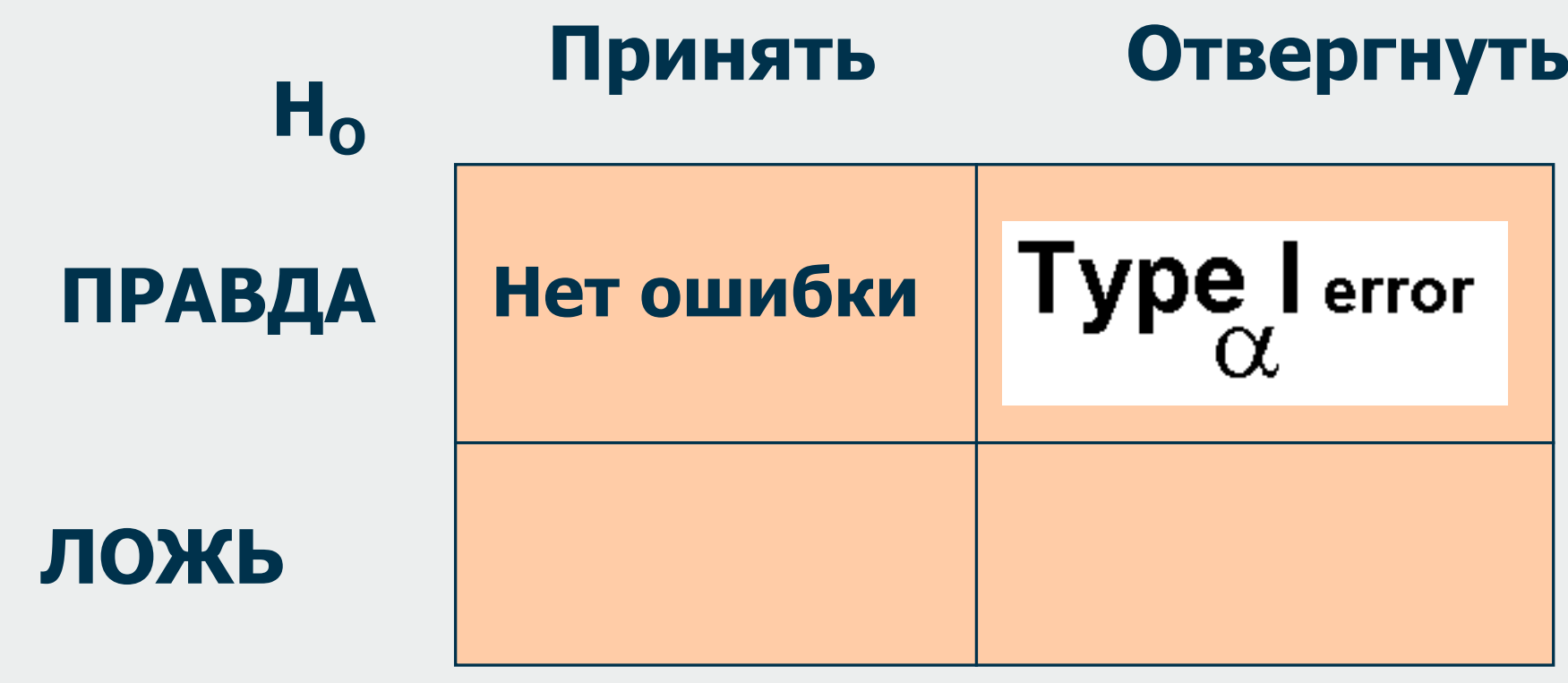

Отвергнуть

Область отклонения

область приема

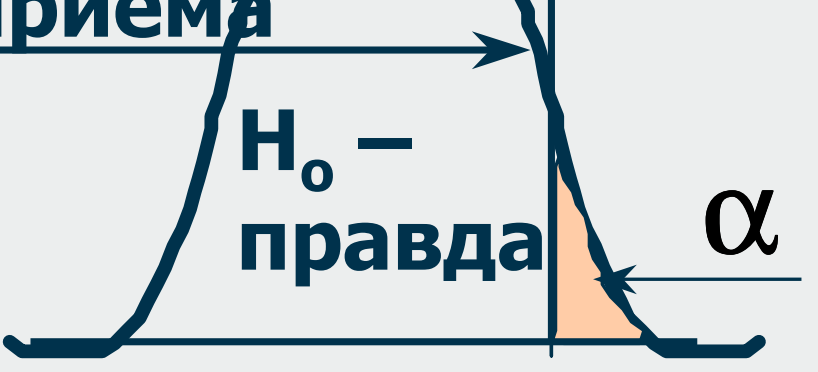




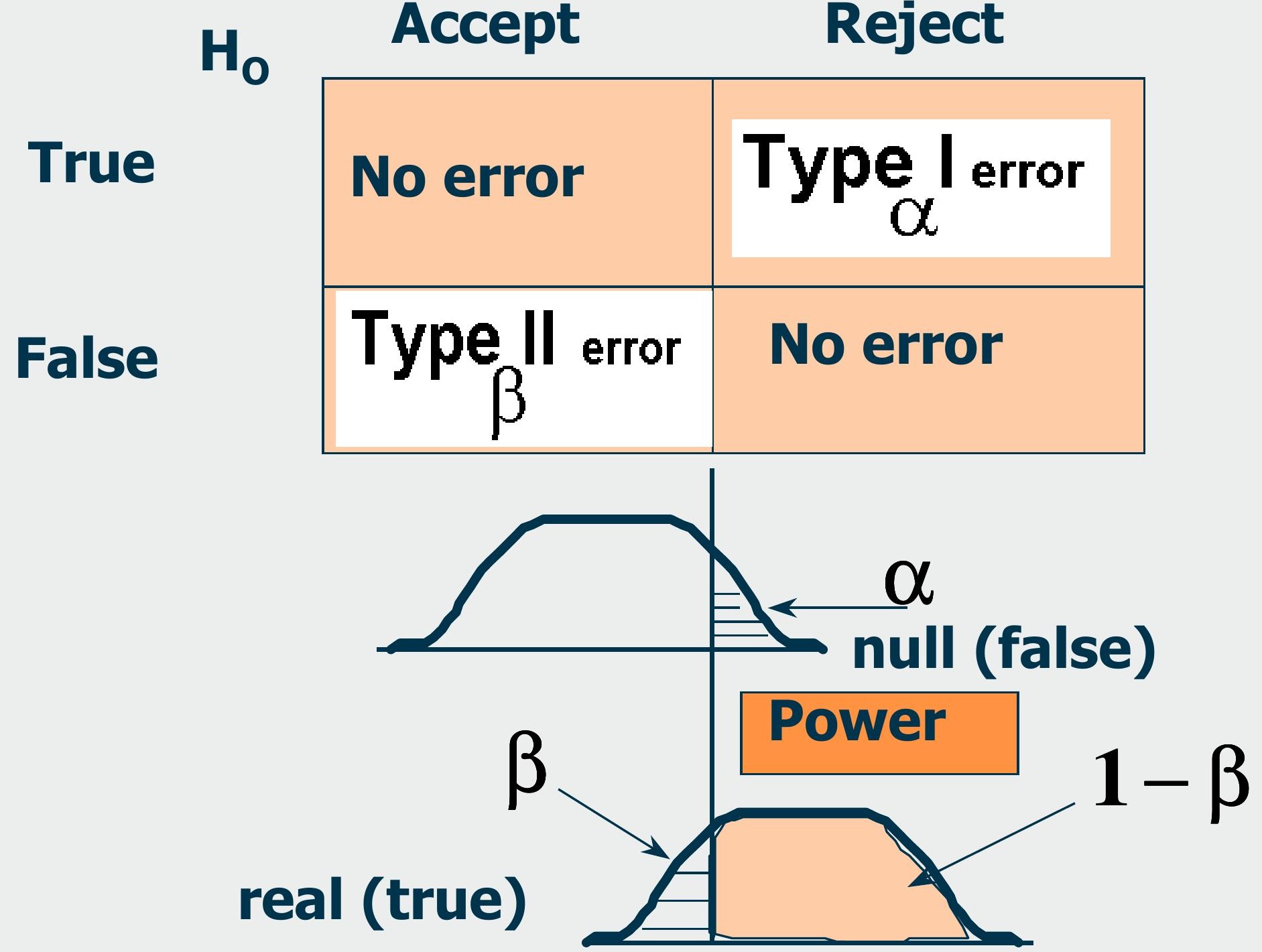




\section{ПОКАЗ ОДНОГО ПРОДУКТА - МОNАDIC ТЕSТ}

- Обычно это лучший из всех методов тестирования продуктов, так как имеет множество достоинств.

1) Monadic тест симулирует реальные жизненные условия, так как обычно в жизни мы употребляем один, а не несколько продуктов в одно и то же время.

2) Исключается взаимовлияние, наблюдаемое при парных сравнениях продуктов.

3) Фокусируя внимание респондента на одном продукте, Monadic тест обеспечивает наиболее точную и действенную диагностическую информацию.

4) Monadic тест обеспечивает использование нормативной базы данных и разработку норм и стандартов действий.

- Теоретически все продукты могут тестироваться в формате Monadic теста, тогда как многие продукты не могут адекватно сравниваться в тестах парных сравнений, например, продукты с очень сильным вкусом (перченые чипсы, алкогольные настойки) могут настолько забить вкус респондента, что он не будет в состоянии тестировать второй продукт. 
ПОСЛЕДОВАТЕЛЬНЫЙ ПОКАЗ ПРОДУКТОВ SEQUENTIAL MONADIC TEST (SM)

- SM очень часто применяется для снижения затрат на проведение теста. В данном формате каждый респондент оценивает два продукта (он или она пробует один продукт и оценивает его, затем пробует второй продукт и оценивает его). SM тест работает достаточно хорошо во многих случаях и имеет ряд тех же достоинств, что и чистый Monadic тест.

Надо всегда помнить об «эфоректе подавления» в данном виде тестов. Все оценки в SM тесте будут ниже по сравнению с чистым Monadic тестом. Следовательно, результаты SM тестов не могут напрямую сравниваться с результатами Monadic тестов. Кроме того, в ряде случаев в SM тестах наблюдается «эрорект взаимовлияния», характерный для парных тестов. Если первый из двух продуктов значительно лучше, тогда второй продукт будет оценен непропорционально низко и наоборот. 


\section{ПРОТОМОНАДИК - PROTOMONADIC TEST}

- Monadic тесты, заканчивающиеся парным сравнением.

- SM тесты, заканчивающиеся парным сравнением.

- Protomonadic тест обеспечивает хорошие диагностические данные, а парное сравнение в конце теста может рассматриваться как подстраховка - как дополнительная гарантия того, что результаты корректны.

Обычно используется в холл-тестах, на не в домашних условиях (из-за сложности исполнения). 


\section{ПAPHOE CPABHEHИE - PAIRED COMPARISON}

- Парное сравнение, при котором респондент пробует два продукта и определяет, какой продукт лучше, обращен к нашему здравому смыслу. Парное сравнение является прекрасным тестом для предъявления доказательств из-за его наглядности. При помощи данного теста возможно измерение очень малых различий между двумя продуктами. Кроме того, парное сравнение часто дешевле других методов, так как во многих случаях размеры выборки меньше.

Тем не менее тест парных сравнений имеет ограниченную ценность для серьезных программ разработки продуктов. Тесты парных сравнений не говорят нам, являются ли оба продукта хорошими или плохими, не могут быть включены в базу нормативных данных. Результаты парных сравнений сильно подвержены эффекту взаимовлияния (например, любые вариации в контрольном продукте вызывают соответствующие вариации в оценках тестового продукта). 
ПОВTOPHOE ПАРHOE CPABHEHИE - REPEATED PAIRS

- B Repeated pairs тестах каждый респондент вначале попарно сравнивает продукт А и Б, а затем эта процедура повторяется.

- Однако при повторном тестировании те же продукты представляют как два других продукта (не как продукты А и Б). Целью Repeated pairs теста является выявление потребителей, не различающих продукты, то есть тех, кто выбрал другой продукт при повторном тесте.

- Считается, что потребители, которые выбрали продукт А в первом тесте и продукт Б во втором тесте, не могут ощутить разницу между этими продуктами. Обычно ответы этих респондентов не учитываются при дальнейших расчетах. Конечные результаты базируются на ответах респондентов, которые различают продукты А и Б, то есть, например, выбравших продукт А в первом и повторном тесте. 
ТЕСТ ТРЕУГОЛЬНИКА - TRIANGLE TEST

- Тест Треугольника используется в первую очередь для тестировании разницы между продуктами. Каждому участнику теста представляются на пробу три продукта и предлагают выбрать один, который отличается от двух других. Тест применяется для определения потребителей, которые способны отличить (идентифицировать) один продукт из трех. В дальнейшем эти респонденты используются В качестве членов небольших экспертных панелей (иногда называемых сенсорными). Используя в дальнейшем Тест Треугольника, эти экспертные панели помогают исследовать и развивать формулы продукта, например, изменения в процессе изготовления, изменения рецептуры и т.д.

- Тест Треугольника используется также для контроля качества (отличий данной партии или продукции различных заводов от стандартной). 


\section{ТЕСТ ДВОЙКА-ТPOЙКА (DUO-TRIO TEST)}

- Одновременно предъявляются три образца

- Один из них помечается как контрольный и трехзначным случайным кодом.

- Два других также помечаются трехзначным случайным кодом.

- Первым тестируется контрольный образец, затем два других. Респондент должен определить образец, отличающийся от контрольного и одинаковый с контрольным.

- План теста может быть:

- С постоянным контрольным образцом

- В этом случае используется один набор кодов для образцов

- Применяется тогда, когда В наличии имеется ограниченное количество одного из образцов

- С равными долями контрольных образцов

- В этом случае используется два набора кодов образцов

- Помогает уменьшить количество тестируемых образцов

- Уменьшает смещение оценок 
- Как предварительная ступень оптимизации продукта или напитка, Ingredient Screening позволяет оценить относительную важность и роль различных компонентов в формуле продукта. Обычно создают несколько формул продукта с высоким и низким уровнем (или отсутствием) конкретного ингредиента. При этом уровни других ингредиентов остаются неизменными. Каждый респондент обычно оценивает от трех до пяти таких формул в зависимости от типа продукта. Продукт оценивается как в целом, так и по конкретным характеристикам (сладость, кислота, текстура, послевкусие).

- Порядок тестирования определяется заранее рассчитанным планом эксперимента. Результаты эксперимента обрабатываются с помощью таких статистических процедур, как ANOVA и MANOVA, а также регрессионным и дискриминантным анализом. 
PRODUCT OPTIMIZATION

- Оптимизация продукта является сложным процессом улучшения продукта, пока он не достигнет максимального уровня потребительской удовлетворенности и степени приятия. Существует множество исследовательских методов, позволяющих найти оптимальный продукт, но термин «Оптимизация продукта» особенно часто применяется к особому структурированному процессу изменения параметров продукта и последующиему тестированию полученных вариантов продукта. Эти варианты оцениваются пользователями товарной категории. Каждый респондент оценивает от трех до пяти вариантов продукта, давая как общую оценку варианта, так и каждой специфической характеристики продукта. Полученные данные анализируются процедурами ANOVA и MANOVA, регрессионным и дискриминантным анализом, а также процедурой поиска экстремумов функции. Результатом является предсказание оптимальной формулы продукта. 
OCHOBHЫE MEPЫ B MONADIC TECTAX

- СТЕПЕНЬ ИНТЕРЕСА К ПОКУПКЕ И ОТКРЫТЫЙ ВОПРОС О ПРИЧИНАХ.

- ОБЩАЯ ОЦЕНКА

- ПОЗИТИВ (ЧТО НРАВИТСЯ, ПРЕИМУЩЕСТВА)

- НЕГАТИВ (ЧТО НЕ НРАВИТСЯ, НЕДОСТАТКИ)

- ЦЕННОСТЬ, УНИКАЛЬНОСТЬ, ПРЕВОСХОДСТВО, ДОВЕРИЕ, ВАЖНОСТЬ

- ОЖИДАЕМАЯ ЧАСТОТА ИСПОЛЬЗОВАНИЯ, КТО ИЗ ЧЛЕНОВ ДОМОХОЗЯЙСТВА ИСПОЛЬЗУЕТ/БУДЕТ ИСПОЛЬЗОВАТЬ

- ОЖИДАЕМАЯ ЧАСТОТА ПОКУПОК, КОЛИЧЕСТВО В ОДНОЙ ПОКУПКЕ

- СИТУАЦИИ ПОТРЕБЛЕНИЯ

- ЗАМЕЩЕНИЕ/ ДРУГОЕ ИСПОЛЬЗОВАНИЕ

- ОЦЕНКА АТРИБУТОВ ПРОДУКТА И ВЫДЕЛЕНИЕ ЕГО СУЩЕСТВЕННЫХ ЧЕPT

- ДЛЯ ДРУГИХ ТЕСТОВ - В СРАВНИТЕЛЬНОЙ ФОРМЕ 
ОБЪЕМ ВЫБОРКИ

КРОМЕ СЛУЧАЕВ НОВЫХ ПРОДУКТОВЫХ КАТЕГОРИЙ (Т.Е. КОГДА ТОВАР СОЗДАЕТ НОВУЮ КАТЕГОРИЮ), ТЕСТИРОВАНИЕ ПРОВОДИТСЯ НА ВЫБОРКАХ СУЩЕСТВУЮЩИХ ПОЛЬЗОВАТЕЛЕЙ, (Е.G., ПОЛЬЗОВАТЕЛИ БЕЗОПАСНЫХ БРИТВ), С РАСШИРЕННЫМИ ГРУППАМИ ДЛЯ КЛЮЧЕВЫХ СЕГМЕНТОВ (Е.G. МУЖЧИНЫ 18-24 ЛЕТ). ОБЩИЙ РАЗМЕР ВЫБОРКИ ДОЛЖЕН ОБЕСПЕЧИТЬ НЕ МЕНЕЕ 100-200 РЕСПОНДЕНТОВ В КАЖДОМ КЛЮЧЕВОМ СЕГМЕНТЕ (ИЛИ НА КАЖДЫЙ ПРОДУКТ ПРИ ПОПАРНОМ СРАВНЕНИИ).

БОЛЬШИЕ РАЗМЕРЫ ВЫБОРОК ПРИВЕСТВУЮТСЯ, ТАК КАК ПОЗВОЛЯЮТ ОБНАРУЖИВАТЬ БОЛЕЕ ТОНКИЕ РАЗЛИЧИЯ МЕЖДУ ПРОДУКТАМИ. МОNADIC - HE MEHEЕ 300!!! 
ЗА И ПРОТИВ

ПОКАЗ ОДНОГО ПРОДУКТА

3А: НАИБОЛЕЕ НЕИСКАЖЕННАЯ ОЦЕНКА ПРОДУКТА С РАСШИРЕННОЙ ДИАГНОСТИКОЙ ЕГО СВОЙСТВ.

ПРОТИВ: БОЛЕЕ ДОРОГИЕ, ИНТЕРПРЕТАЦИЯ ЗАТРУДНЕНА ПРИ ОТСУТСТВИИ КОНТРОЛЬНОГО ПРОДУКТА ИЛИ НОРМАТИВНОЙ БАЗЫ ТЕСТОВ.

СРАВНИТЕЛЬНЫЕ

3А: МЕНЬШЕ ЦЕНА И ОБЪЕМЫ ВЫБОРКИ ПРИ СРАВНЕНИИ БОЛЬШОГО КОЛИЧЕСТВА ПРОДУКТОВ

ПРОТИВ: ОГРАНИЧЕННАЯ ДИАГНОСТИКА СВОЙСТВ, ОТНОСИТЕЛЬНЫЙ ХАРАКТЕР ОЦЕНОК (НЕ КАК В РЕАЛЬНОЙ ЖИЗНИ). 
СЛЕПЫЕ ТЕСТЫ

ГЛАВНОЙ ЦЕЛЬЮ СЛЕПЫХ ТЕСТОВ ЯВЛЯЕТСЯ ПОЛУЧЕНИЕ «ГЕДОНИСТИЧЕСКОЙ ОЦЕНКИ» ПРОДУКТА. ФОКУСИРОВКА НА:

- ОСНОВНЫХ/БАЗОВЫХ ХАРАКТЕРИСТИКАХ ОЦЕНКИ ПРОДУКТА

- ИЗБЕГАНИЕ СРАВНЕНИЯ ОДИНАКОВО ГЕДОНИСТИЧЕСКИ ОЦЕНИВАЕМЫХ, НО ПО РАЗНОМУ ВОСПРИНИМАЕМЫХ ПРОДУКТОВ;

- ПОЛУЧЕНИЕ ТЕСТОВЫХ БАЗ ДАННЫХ, ПОЗВОЛЯЮЩИХ ОЦЕНИТЬ ИЗМЕНЕНИЕ ПРОДУКТА НА БАЗЕ ВНУТРЕННЕ ПРИСУЩИХ ЕМУ ХАРАКТЕРИСТИК (Т.е. НЕИМИДЖЕВЫХ) 
TECT-MAPKET FMCG (DISPOSABLE TEST MARKET)

ПРОГРАММА ТЕСТА

ВЫБОРКА - ТЕСТОВЫЕ ГОРОДА И ТОРГОВЫЕ ТОЧКИ

ВЫБОР СПИСКА МАРОК (BENCHMARK BRANDS)

РЕКРУТИРОВАНИЕ ТОРГОВЫХ ТОЧЕК

ОПРЕДЕЛЕНИЕ ОБЪЕМА ПРОДАЖИ БЕНЧМАРОК (ПРЕ-ТЕСТ)

ИДЕНТИФИКАЦИЯ ПОКУПАТЕЛЕЙ (ЦГ)

РЕКРУТИРОВАНИЕ ПАНЕЛИ ( 2000 РЕСПОНДЕНТОВ - «ПАНЕЛИСТОВ»)

РАЗМЕЩЕНИЕ ТЕСТ ПРОДУКТА НА ПОЛКАХ

ПОЧТОВАЯ РАССЫЛКА ИЗВЕЩЕНИЯ «ПАНЕЛИСТАМ» (ОПЦ. - КУПОН)

ЕЖЕНЕДЕЛЬНЫЕ ОБЪЕМЫ ПРОДАЖ ПО БЕНЧМАРКАМ И ТЕСТ ПРОДУКТУ

ОБЗВОН «ПАНЕЛИСТОВ»

ПОВТОРНЫЙ ОБЗВОН «ПАНЕЛИСТОВ» 
TECT-MAPKET FMCG (DISPOSABLE TEST MARKET)

РЕЗУЛЬТАТЫ ТЕСТА

ЕЖЕНЕДЕЛЬНЫЙ ОБЪЕМ ПРОДАЖ

ДОЛЯ ПРОДАЖ ТЕСТ-ПРОДУКТА ОТНОСИТЕЛЬНО ВЫБРАННЫХ БЕНЧМАРОК

УРОВЕНЬ ПОВТОРНЫХ ПОКУПОК

ИСПОЛЬЗОВАНИЯ ТАЛОНОВ

ОЦЕНКА ТЕСТ-ПРОДУКТА ПОКУПАТЕЛЯМИ

СТЕПЕНЬ СООТВЕТСТВИЯ ТЕСТ-ПРОДУКТА ОЖИДАНИЯМ

ПОЧЕМУ ПРОДУКТ (НЕ-)СООТВЕТСТВУЕТ ОЖИДАНИЯМ

ПРИЧИНЫ ОТКАЗА ОТ ПОКУПКИ

ЦЕНА ТЕСТ-МАРКЕТА НА 2000 РЕСПОНДЕНТАХ ПРИМЕРНО ТАКАЯ ЖЕ, КАК ТЕСТИРОВАНИЕ ПРОДУКТА НА 500-600 РЕСПОНДЕНТАХ, ОДНАКО РЕЗУЛЬТАТЫ ПОЛУЧАЮТСЯ НЕ НА «ТЕСТЕРАХ», А РЕАЛЬНЫХ ПОКУПАТЕЛЯХ. 
HOME versus HALL TECTЫ

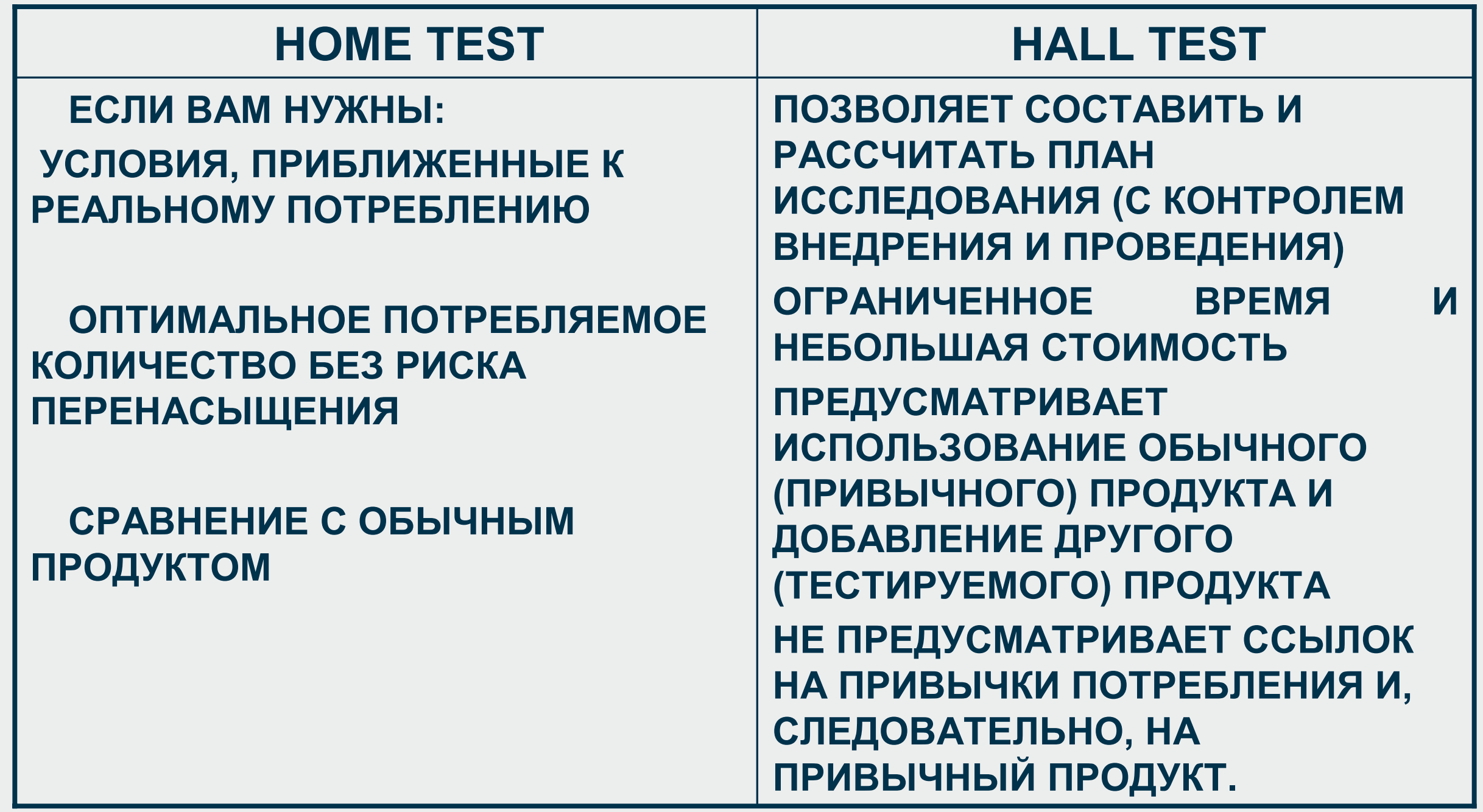




\section{ПОРЯДОК ПРЕДЪЯВЛЕНИЯ ОБРАЗЦОВ}
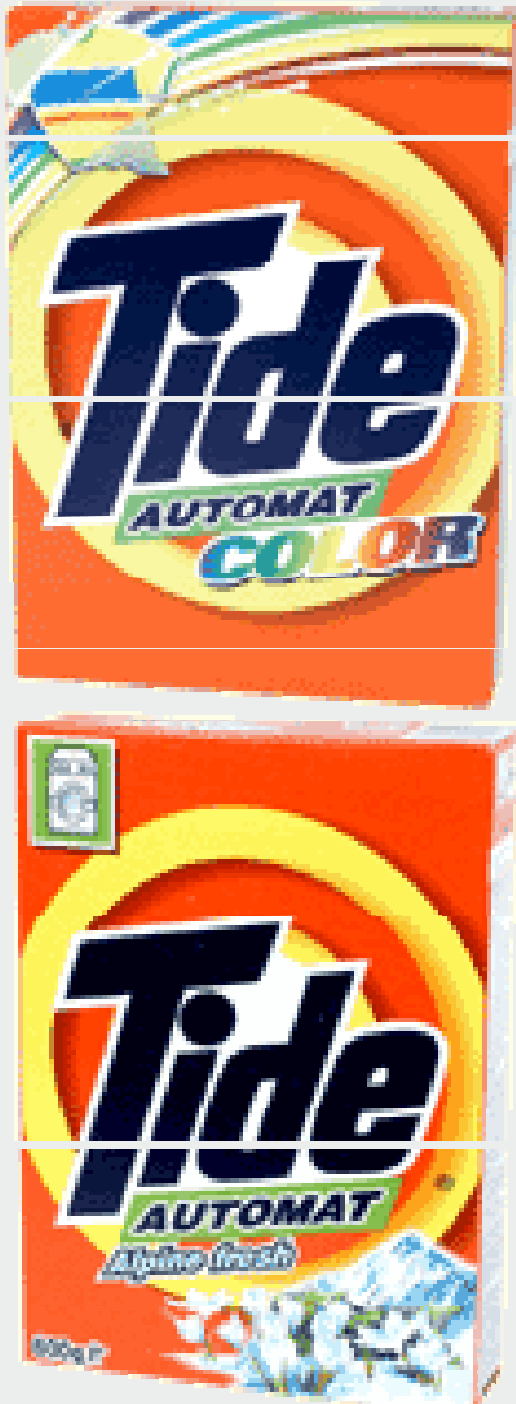

- ДВА ЭФФЕКТА ВЛИЯНИЯ ПОРЯДКА:

ЕСЛИ НЕЗАВИСИМО ОТ ПОРЯДКА ПРИ НАЛИЧИИ СДВИГА ПРЕДПОЧТЕНИЙ К ПЕРВОМУ ПО ПОРЯДКУ ПРЕДЪЯВЛЕНИЯ ОБРАЗЦУ, ТО ЭТО ОЗНАЧАЕТ, ЧТО ОБА ПРОДУКТА ХОРОШО ПРИНИМАЮТСЯ РЫНКОМ. И НАОБОРОТ, ЕСЛИ НЕЗАВИСИМО ОТ ПОРЯДКА ПРИ НАЛИЧИИ СДВИГА ПРЕДПОЧТЕНИЙ КО ВТОРОМУ ПО ПОРЯДКУ ПРЕДЪЯВЛЕНИЯ ОБРАЗЦУ, ТО ЭТО ОЗНАЧАЕТ, ЧТО ОБА ПРОДУКТА НЕ ЛУЧШЕ ИЛИ ХУЖЕ ИМЕЮЩИХСЯ НА РЫНКЕ.

РЕЗУЛЬТАТЫ ТЕСТИРОВАНИЯ МОГУТ ЗАВИСЕТЬ ОТ ПОРЯДКА ТЕСТИРОВАНИЯ. ЭТО СЛУЧАЕТСЯ В ТОМ СЛУЧАЕ, ЕСЛИ ОДИН ИЗ ПРОДУКТОВ ИМЕЕТ ЯВНОЕ ПРЕИМУЩЕСТВО В ХАРАКТЕРИСТИКАХ. И НЕ ВСЕГДА ЭТО КОНТРОЛИРУЕМЫЙ В ТЕСТЕ ПАРАМЕТР, НАПРИМЕР, ЭТО МОГУТ БЫТЬ ТАКТИЛЬНЫЕ ОЩУЩЕНИЯ ОТ РАЗНЫХ УПАКОВОК.

- ПРИМЕР

- ЕСЛИ СНАЧАЛА ТЕСТИРОВАТЬ ТIDE ОБЫЧНЫЙ, А ЗАТЕМ TIDE С ОТБЕЛИВАТЕЛЕМ, ТО РАЗНИЦА МЕЖДУ ПОРОШКАМИ ОЧЕНЬ ЗАМЕТНА. ЕСЛИ ПОРЯДОК ТЕСТИРОВАНИЯ ОБРАТНЫЙ, ТО УДАЛЕНИЕ ПЯТЕН И ОКРАШИВАНИЯ ТКАНИ ПОСЛЕ ПЕРВОГО ТЕСТА ДЕЛАЮТ РАЗНИЦУ МЕЖДУ ПОРОШКАМИ НЕ СТОЛЬ ЗАМЕТНОЙ. 


\section{ПОРЯДОК ПРЕДЪЯВЛЕНИЯ ОБРАЗЦОВ (ПРИМЕР)}

- ЕСЛИ СНАЧАЛА ТЕСТИРОВАТЬ ТIDЕ ОБЫЧНЫЙ, А ЗАТЕМ ТІDЕ С ОТБЕЛИВАТЕЛЕМ, ТО РАЗНИЦА МЕЖДУ ПОРОШКАМИ ОЧЕНЬ ЗАМЕТНА. (ПРЕВОСХОДСТВО ДОСТИГАЕТ СООТНОШЕНИЯ 80/20).

- ЕСЛИ ПОРЯДОК ТЕСТИРОВАНИЯ ОБРАТНЫЙ, ТО УДАЛЕНИЕ ПЯТЕН И ОКРАШИВАНИЯ ТКАНИ ПОСЛЕ ПЕРВОГО ТЕСТА ДЕЛАЮТ РАЗНИЦУ МЕЖДУ ПОРОШКАМИ НЕ СТОЛЬ ЗАМЕТНОЙ. (СООТНОШЕНИЕ ПАДАЕТ ДО 55/45).
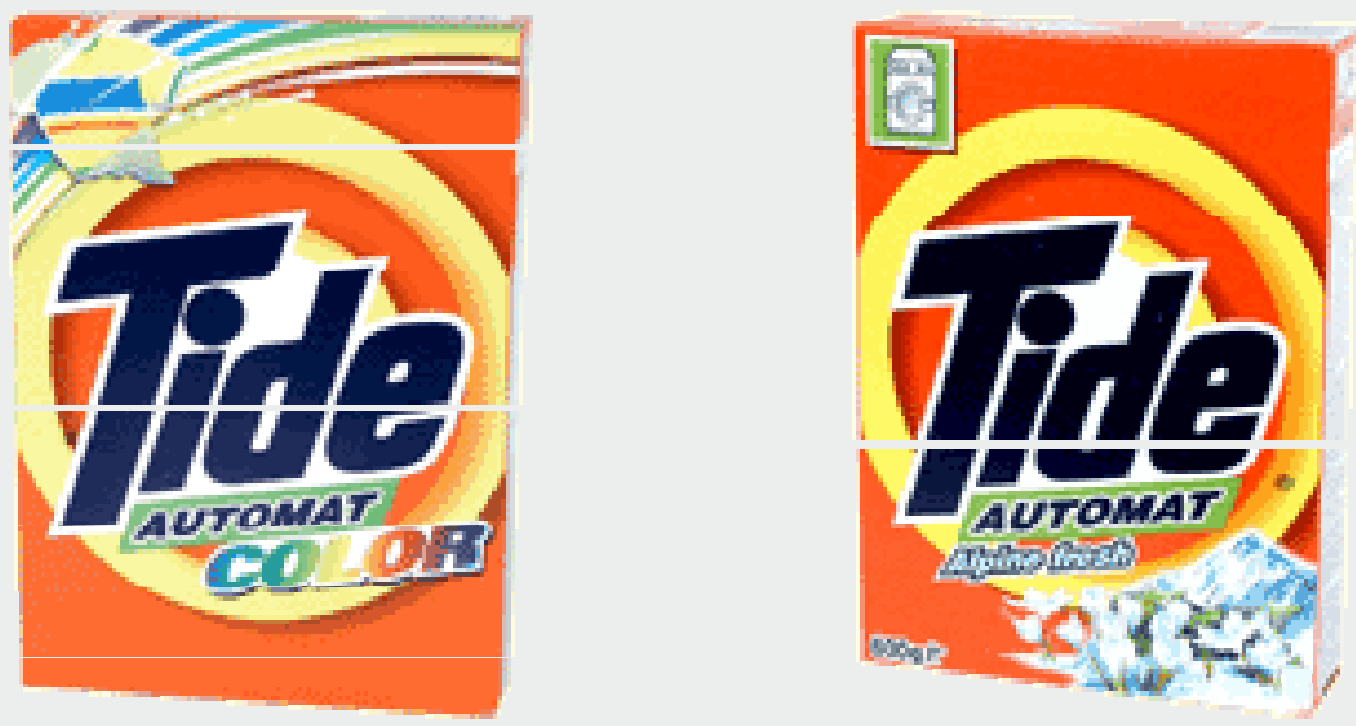


\section{ПОРЯДОК ПРЕДЪЯВЛЕНИЯ ОБРАЗЦОВ ПPИMEP SEQUENTIONAL MONADIC}

ИССЛЕДОВАНИЯ, ПРОВЕДЕННЫЕ Р\&G, ПОКАЗАЛИ, ЧТО В ડМ ТЕСТАХ ПОРЯДОК ОКАЗЫВАЕТ СИЛЬНОЕ ВЛИЯНИЕ НА ОЦЕНКУ.

\begin{tabular}{|c|c|c|c|c|}
\hline \multicolumn{2}{|c|}{ Product } & Base & \multicolumn{2}{|l|}{ Ratings } \\
\hline & & Used & Used & Average \\
\hline & & First & Second & \\
\hline$A$ & 163 & 6.84 & 6.25 & 6.55 \\
\hline$B$ & 159 & 6.53 & 6.48 & 6.50 \\
\hline$C$ & 156 & 6.51 & 6.38 & 6.44 \\
\hline$D$ & 162 & 6.89 & 6.44 & 6.66 \\
\hline \multicolumn{2}{|c|}{ Range } & .38 & .23 & .22 \\
\hline
\end{tabular}

КРОМЕ ТОГО ВЫЯСНИЛОСЬ, ЧТО ВОПРОСЫ, ЗАДАННЫЕ ПРО ОТДЕЛЬНЫЕ ХАРАКТЕРИСТИКИ ПЕРВОГО ПО ПОРЯДКУ ПРОДУКТА, ТАКЖЕ ОКАЗЫВАЮТ ВЛИЯНИЕ НА ОЦЕНКУ ВТОРОГО ПО ПОРЯДКУ ПРОДУКТА.

ПОПЫТКА ОБЪЕДИНИТЬ В SМ ТЕСТАХ ДОСТОИНСТВА MONADIC И PAIR COMPARISON ПРОЦЕДУР ПРИВЕЛА К ОБРАТНОМУ ЭФФЕКТУ. 
